\title{
Nanofibrous Synthetic Dural Patch for Skull Base Defects: Preliminary Experience for Reconstruction after Extended Endonasal Approaches
}

\author{
Francesco Zenga ${ }^{1}$ Valentina Tardivo ${ }^{1}$ Paolo Pacca ${ }^{1}$ Massimiliano Garzaro ${ }^{2}$ Diego Garbossa ${ }^{1}$ \\ Alessandro Ducati ${ }^{1}$ \\ ${ }^{1}$ Division of Neurosurgery, Department of Neuroscience, University of \\ Torino, Turin, Italy \\ 21 st Division of ENT, Department of Surgical Sciences, University of \\ Torino, Turin, Italy \\ Address for correspondence Valentina Tardivo, MD, Division of \\ Neurosurgery, Department of Neuroscience, University of Torino, \\ Turin, Italy (e-mail: tardivo.valentina@gmail.com).
}

J Neurol Surg Rep 2016;77:e50-e55.

\author{
Abstract \\ Keywords \\ - extended endoscopic \\ endonasal approach \\ - cerebrospinal fluid \\ leak \\ - dural substitute
}

Setting One of the consequences of the widespread use of endoscopic endonasal approaches (EEA) to skull base pathologies is the management of complex skull base defects. Nowadays, the gold standard is a multilayer closure that reproduces the physiological tissue barriers. Several techniques have been described in the literature; however, skull base reconstruction after EEA still represents a matter of debate, especially after extended EEA. A watertight closure is paramount to prevent cerebrospinal fluid leak and meningitis.

Design Regarding this issue, we present our experience with a new synthetic dural patch, ReDura (Medprin Biotech, La Mirada, California, United States), as a subdural inlay in three patients who underwent endoscopic endonasal removal of sellar and suprasellar lesions.

Conclusions ReDura patch showed the same versatility of autologous iliotibial tract. A dural patch that easily adapts to all defects, revealed to be a useful tool for performing watertight closure, possibly in a short operative time, after endoscopic approaches.

\section{Introduction}

Endoscopic endonasal approach (EEA) is nowadays the gold standard in the treatment of most sellar and suprasellar lesions. Endoscopy in fact provides direct access to midline structure without needing brain retraction and minimizing opening of the surface, while maximizing resection closer to the target. ${ }^{1}$ Cooperation between otolaryngologists and neurosurgeons, technological improvements, and the development of dedicated instruments greatly contributed to the reproducibility and success of the endoscopic endonasal technique.

received

March 29, 2015

accepted after revision

November 7, 2015
DOI http://dx.doi.org/ $10.1055 / \mathrm{s}-0035-1570388$. ISSN 2193-6358.
However, reconstruction of the barriers between the intracranial space and the sinonasal tract continues to be a challenge after expanded endonasal cranial base surgery. As a matter of fact, cerebrospinal fluid (CSF) leak and, eventually, a subsequent meningitis are still a concern.

Usually, tissue barrier is reestablished with a multilayered reconstruction. In our department, small CSF leaks in sellar surgeries are closed using fat over the dural defect and fibrin glue. When dealing with large dural defects, iliotibial tract (ITT) is usually placed as an inlay intradural graft. The HadadBassagasteguy septal mucosal flap, ${ }^{2}$ previously harvested, is laid down over the surgical defect and abdominal free fat may

\footnotetext{
(c) 2016 Georg Thieme Verlag KG Stuttgart · New York
}

License terms

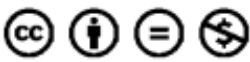


be also employed to obliterate the dead spaces. As previously highlighted, ${ }^{3}$ (ITT) has favorable features in terms of strength, pliability, thickness, availability, and biocompatibility. However, its harvesting leads to a longer operative time and higher risk of infection, and causes an external scar. According to these limitations of ITT, we found ReDura, a new nanofibrous synthetic dural patch by Medprin Biotech, a reliable alternative option to ITT. In fact, ReDura showed similar manageability as ITT without the risk of muscle prolapse, with a better aesthetic result.

In this technical report, we describe our preliminary experience with ReDura in three cases of skull base repair after an extended EEA. Two of these cases are reported and described with intraoperative pictures and magnetic resonance imaging (MRI) scans.

\section{Case Report 1}

We report a case of a 50-year-old woman with a midline skull base cystic lesion with microcalcification diagnosed with a head computed tomography (CT) after syncope. Since her childhood she had right eye visual impairment due to an inflammatory disease. However, the patient complained an acute worsening of the visual deficit in the last months before the diagnosis.

The MRI scan confirmed the presence of a sellar-suprasellar cystic mass that failed to enhance with contrast, causing left deviation of the pituitary stalk and imprinting the optic chiasm (-Fig. 1).

Patient complained of recurrent headache refractory to medical therapy starting 6 months before diagnosis. The endocrinologic evaluation revealed no pituitary hormones abnormality. The ophthalmologic assessment found visual acuity impairment in the right eye, while no visual field impairment was detected. An extended endoscopic endonasal transtubercular approach was employed to expose the suprasellar cyst. The classic "two nostrils four hands" surgical

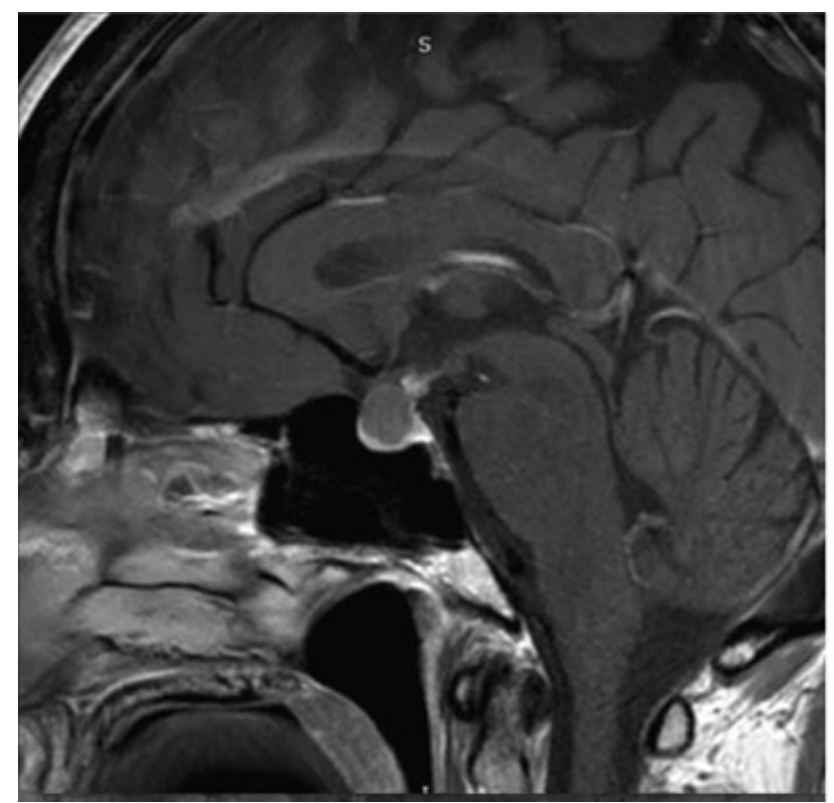

technique originally described by Kassam et al was used. ${ }^{4}$ Surgical approach is summarized in - Fig. 2. The postoperative course was regular without new onset of neurological deficit and without sign or symptoms of CSF leak.

The histological exam revealed that the lesion was a Rathke cyst cleft. The 3-month postoperative MRI confirms the physiological healing of the surgical dural defect and the partial degradation of the dural patch (-Fig. 3).

\section{Case Report 2}

A 50-year-old woman who underwent two microscopic transsphenoidal intervention, Gamma Knife radiosurgery, and microsurgical removal via pterional approach of a recurrent clival chordoma since 2008 complained a new onset of right temporal hemianopsia in September 2014. A brain MRI showed a volumetric increase of the residual chordoma compressing and dislocating the optic chiasm (-Fig. 4).

The patient underwent an extended endoscopic endonasal removal of the tumor. A transtubercular-transplanum-transclival approach was chosen.

Then a multilayer closure was performed: abdominal fat, taken from infraumbilical incision, was placed in the surgical cavity as first step, fibrin glue was then employed, and ReDura patch was used as inlay subdural graft. The HadadBassagasteguy septal mucosal flap previously harvested was laid down over the surgical defect (-Fig. 5). Fibrin sponge was positioned over the flap and nasal "glovefinger-fill with polyvinyl alcohol sponge" tampons were then placed in both nostrils to improve watertight closure. No sign or symptom of CSF leak or meningitis was seen during the hospital stay or after the discharge.

One month postoperative, MRI confirms the absence of any leak and show the remnants of ReDura as inlay subdural graft (-Fig. 6).

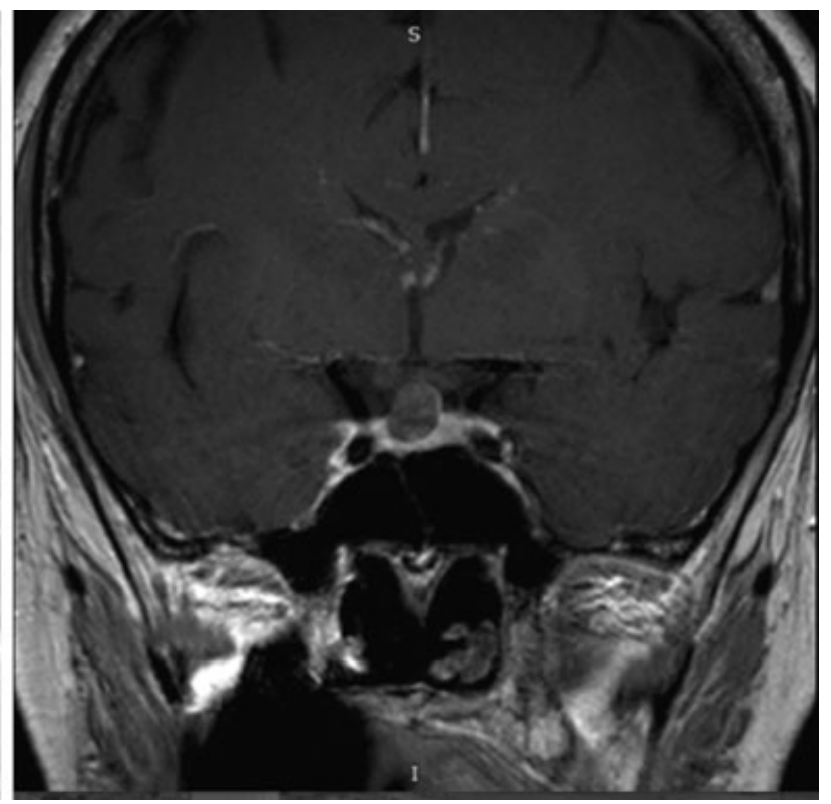

Fig. 1 Case 1: Preoperative MRI. 


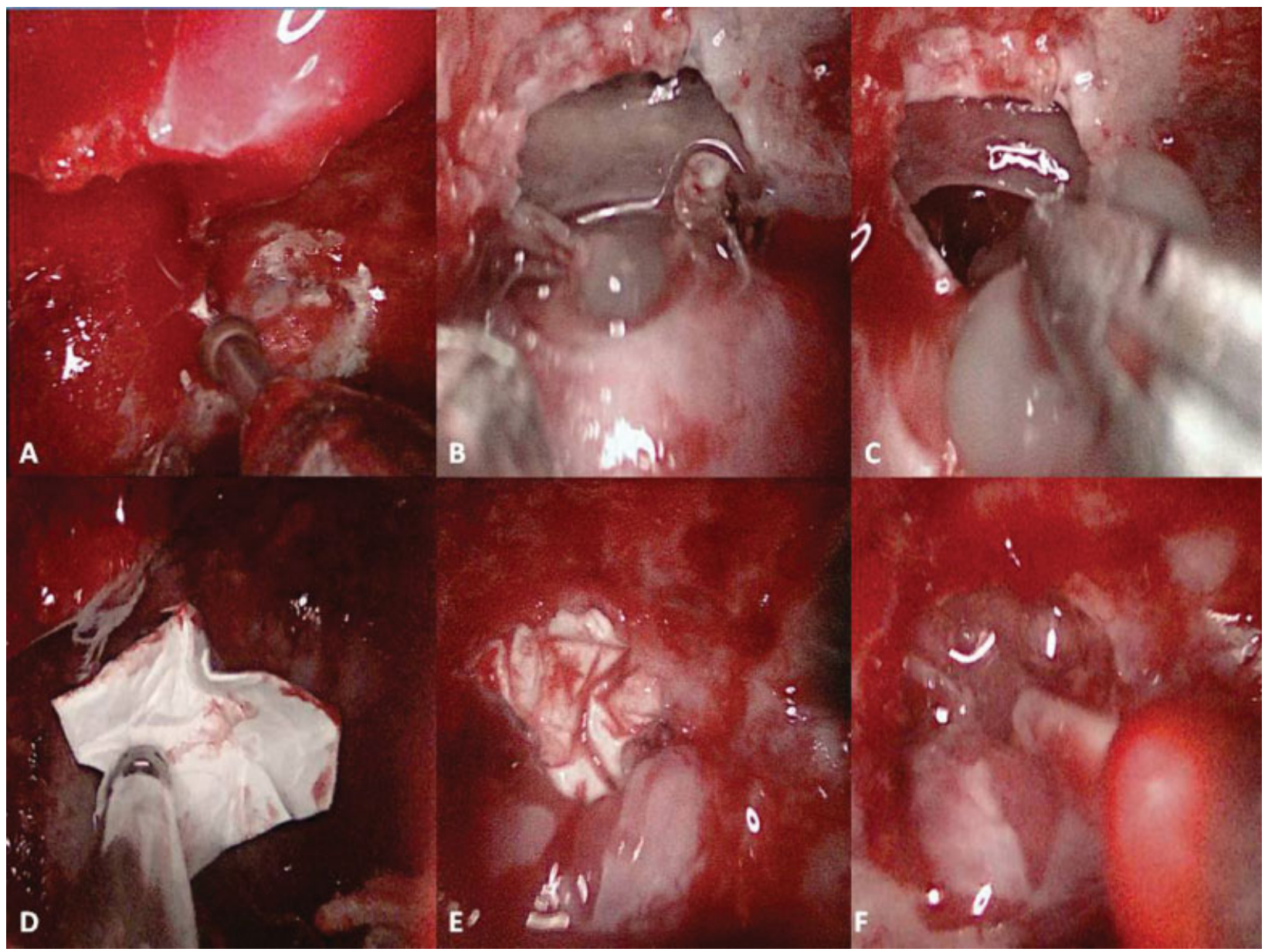

Fig. 2 (A) Drilling of the tuberculum sellae. (B) Dural exposition. (C) Cystic lesion removal. (D) Application of ReDura. (E) ReDura used as subdural inlay. (F) Fibrin glue over the dural patch.
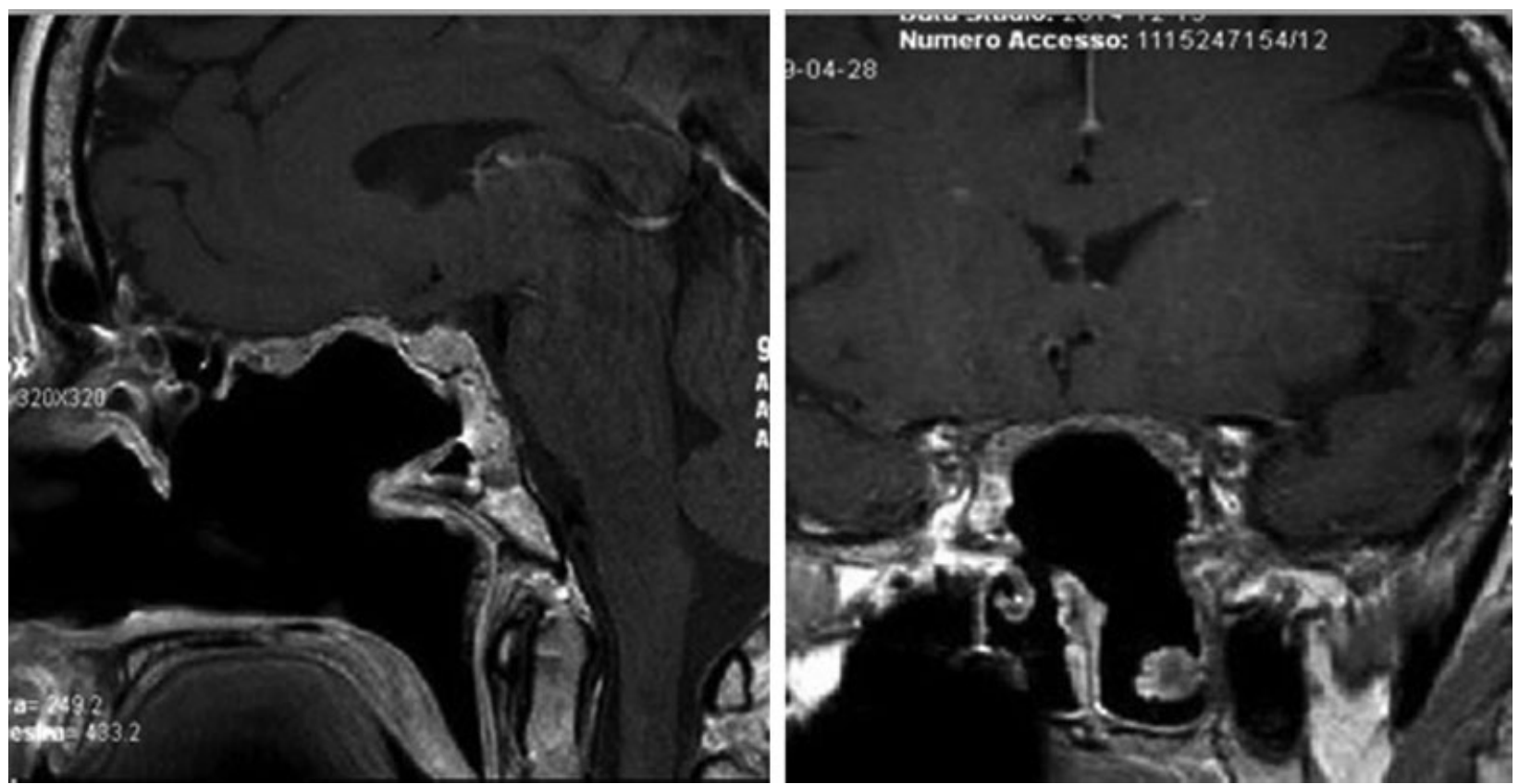

Fig. 3 Case 1: 3-month postoperative gadolinium-enhanced brain MRI: watertight closure, partial degradation of ReDura. 


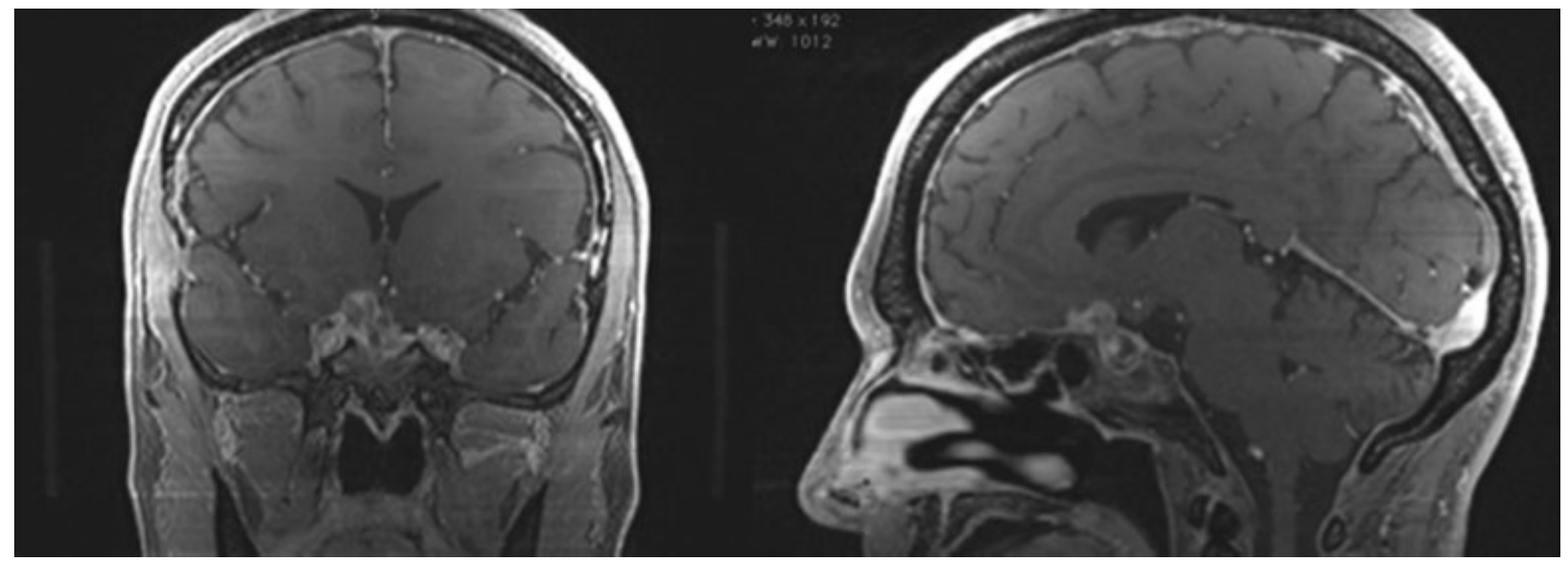

Fig. 4 Case 2: Preoperative MRI.

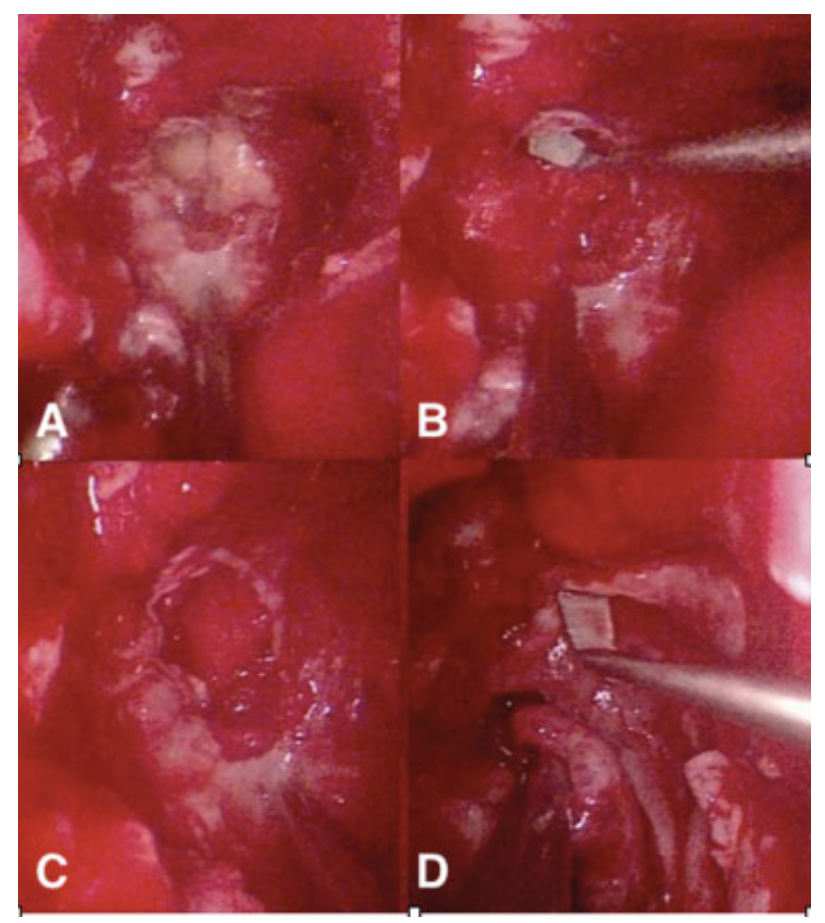

Fig. 5 Multilayer closure. (A) Autologous abdominal fat in the surgical cavity to fill dead spaces. (B, C) ReDura as subdural inlay. (D) Retrieving Hadad vascularized nasoseptal flap over the surgical defect.

\section{Case Report 3}

A 61-year-old man underwent brain MRI after a syncope episode and hypertensive crisis, and showed a sellar-suprasellar cystic lesion causing pituitary gland compression. Peripheral intralesional calcifications were revealed by a brain CT. Both physical examination and laboratory test excluded endocrinological disorders. No visual field deficits or visual impairment were detected. Endoscopic endonasal transsphenoidal removal of the lesion was performed. Multilayer closure was done using ReDura as subdural inlay, as previously described. No sign or symptom of CSF leak was detected during the hospital stay or at the discharge. The histological examination revealed a craniopharyngioma.

\section{Discussion}

Despite the technical advances in EEA, closure of dural defect is still challenging and postoperative CSF leak is still a concern. A recent review by Soudry et al reports that the overall postoperative CSF leak rate is $8.5 \%$, regardless of reconstructive technique. ${ }^{5}$

As highlighted by several authors, ${ }^{6-8}$ the development of reconstructive techniques to ensure watertight closure to prevent infection and to promote dura regeneration is paramount. Multilayer reconstruction made with mucosal flap and sponges is mandatory in cases of large skull base defects. Related to this issue, we describe the first attempt to use a synthetic nanofibrous dural patch in multilayer reconstruction after EEA.

Healing of dural defect is due to fibroblastic proliferation and their ability to produce new collagen and connective tissue. Synthetic dural patches seem to act as a scaffold for dural cells migration. When a degradable patch is used, dural repair is thought to be related to the replacement of the graft by endogenous tissue. ${ }^{9}$

ReDura is regenerative dural repair patch made of synthetic absorbable biomaterial scaffold resembling microstructure of native dural matrix that provides appropriate environment for recruitment, growth, and proliferation of dural cell on scaffolds. ReDura is made of poly-L-lactide (PLLA) based on biomimetic electrospun technology. Electrospinning has recently emerged as a potential method in manufacturing nano- or micro-sized fibers from synthetic or natural polymers. Such fibers can mimic the morphology and physical structure of extracellular matrix components to promote cell adhesion and growth. ${ }^{10}$ This new product proved to be safe and efficacious in animal and clinical research. $^{11}$

ReDura graft, used as an inlay in the subdural space, seems to offer several advantages in skull base reconstruction after EEA, in terms of versatility, biocompatibility, and promoting dura regeneration.

Once rehydrated, this patch reach the same consistency of a muscular fascia, soft, flat, and easy to handle, that we found to be very suitable to manage all dural defects' conformations. 


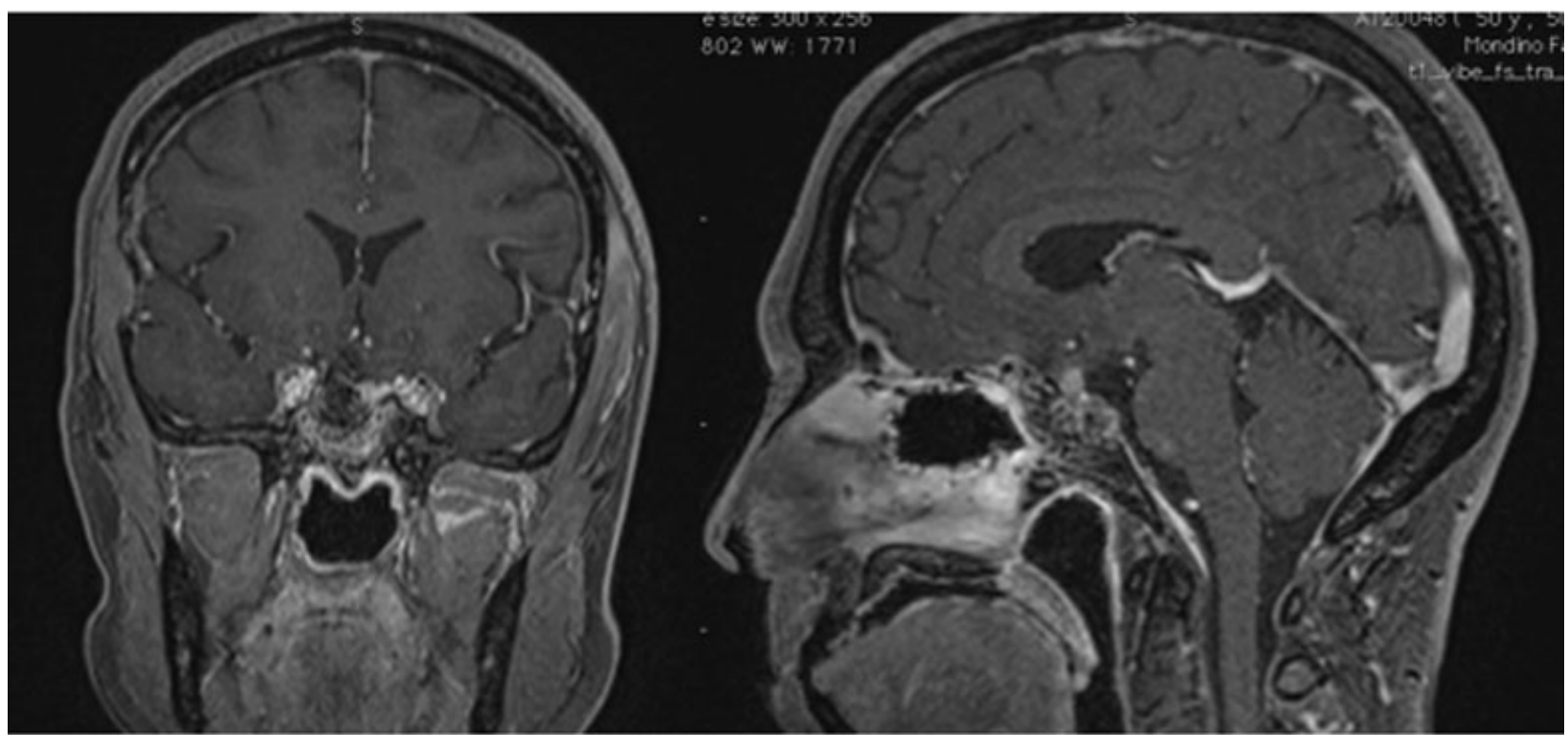

Fig. 6 One-month postoperative brain MRI after recurrent clival chordoma removal. Multilayer skull base reconstruction is possible to appreciate the fat placed in the surgical cavity to obliterate dead space and the ReDura patch as subdural Inlay.

As reported by Villaret et al, ITT is the ideal graft for duroplasty thank to its thickness, pliability, and strength. ${ }^{3}$ ReDura patch showed the same versatility as autologous iliotibial tract. However, the use of ReDura instead of ITT prevents an external scar in the area when ITT is usually harvested.

A dural patch that easily adapts to all defects, revealed to be a useful tool for performing watertight closure, possibly in a short operative time, after endoscopic approaches.

Moreover, the potential of ReDura to promote dural regeneration, described in both animal and clinical studies, results very interesting. This is related to its 3D nanofibrous structure that provides a network for cells migration into scaffold, improving tissue reconstructions. Preclinical animal studies reported that 3 months after ReDura implantation, newborn membrane in defect and scaffold material was mostly degraded. Besides, rapid growth of neodural tissue in patient where ReDura was used as a dural patch after neurosurgical open procedures was reported. One week after surgery, head CT demonstrates initial dural regeneration. ${ }^{11}$

After 2 to 3 months, the ReDura PLLA material itself degraded, undergoing full loss of its mechanical properties and integrity, and the entire patch was converted to a high number of PLLA tiny defragments, which subsequently underwent complete degradation. Compared with other synthetic dural patches, ReDura completely degraded in few months, reducing the risk of infection associated with foreign bodies. $^{11}$

\section{Conclusion}

As previously pointed out by Tabaee et $\mathrm{al}^{12}{ }^{12}$ the best graft for duraplasty should be easy to harvest, available in adequate quantity, biocompatible with a minimal risk of rejection, and associated with minimal morbidity. ReDura PLLA mesh man- ufactured by electrospinning was first applied as a dural substitute in endoscopic endonasal surgery. We are cautiously optimistic that ReDura have all these features. Further studies on patients are needed to attest the advantages of the new synthetic dural patches, together with a cost-effectiven evaluation.

\section{References}

1 Koutourousiou M, Gardner PA, Fernandez-Miranda JC, Paluzzi A, Wang EW, Snyderman $\mathrm{CH}$. Endoscopic endonasal surgery for giant pituitary adenomas: advantages and limitations. J Neurosurg 2013;118(3):621-631

2 Hadad G, Bassagasteguy L, Carrau RL, et al. A novel reconstructive technique after endoscopic expanded endonasal approaches: vascular pedicle nasoseptal flap. Laryngoscope 2006;116(10): 1882-1886

3 Villaret AB, Schreiber A, Battaglia P, Bignami M. Endoscopyassisted iliotibial tract harvesting for skull base reconstruction: feasibility on a cadaveric model. Skull Base 2011;21(3):185-188

4 Kassam A, Snyderman CH, Mintz A, Gardner P, Carrau RL. Expanded endonasal approach: the rostrocaudal axis. Part I. Crista galli to the sella turcica. Neurosurg Focus 2005;19(1):E3

5 Soudry E, Turner JH, Nayak JV, Hwang PH. Endoscopic reconstruction of surgically created skull base defects: a systematic review. Otolaryngol Head Neck Surg 2014;150(5):730-738

6 Paluzzi A, Gardner P, Fernandez-Miranda JC, Snyderman C. The expanding role of endoscopic skull base surgery. Br J Neurosurg 2012;26(5):649-661

7 Malliti M, Page P, Gury C, Chomette E, Nataf F, Roux FX. Comparison of deep wound infection rates using a synthetic dural substitute (neuro-patch) or pericranium graft for dural closure: a clinical review of 1 year. Neurosurgery 2004;54(3):599-603, discussion 603-604

8 Thorp BD, Sreenath SB, Ebert CS, Zanation AM. Endoscopic skull base reconstruction: a review and clinical case series of 152 vascularized flaps used for surgical skull base defects in the setting of intraoperative cerebrospinal fluid leak. Neurosurg Focus 2014;37(4):E4 
9 Weber R, Keerl R, Draf W, Schick B, Mosler P, Saha A. Management of dural lesions occurring during endonasal sinus surgery. Arch Otolaryngol Head Neck Surg 1996;122(7):732-736

10 Kurpinski K, Patel S. Dura mater regeneration with a novel synthetic, bilayered nanofibrous dural substitute: an experimental study. Nanomedicine (Lond) 2011;6(2):325-337
11 Shi ZD, Xu T, Yuan YY, et al. Use of novel regenerative nanofibrous bio-device for dural defect repair. Paper presented at: EANS/ The14th European Congress of Neurosurgery; 2011; Rome, Italy

12 Tabaee A, Anand VK, Brown SM, Lin JW, Schwartz TH. Algorithm for reconstruction after endoscopic pituitary and skull base surgery. Laryngoscope 2007;117(7):1133-1137 